Pacific Journal of Mathematics

ON WEAK EPIMORPHISMS IN HOMOTOPY THEORY 


\title{
ON WEAK EPIMORPHISMS IN HOMOTOPY THEORY
}

\author{
JOSEPH ROITBERG
}

\begin{abstract}
Weakened versions of the categorical notions of epimorphism and monomorphism have proved to be of some interest in pointed homotopy theory. A weak epimorphism, for instance, is a morphism $e$ (in any category with 0 objects) such that $g \circ e=0$ implies $g=0$.

In 1967, Ganea utilized extensive homotopy-theoretic calculations to exhibit examples, in the pointed homotopy category, of weak monomorphisms which are not monomorphisms. In this note, we exploit the properties of a remarkable group discovered by Higman in 1951 to exhibit examples, again in the pointed homotopy category, of weak epimorphisms which are not epimorphisms, thereby confirming a suspicion enunciated by Hilton in the early 1960's.
\end{abstract}

1. A weak epimorphism in a category with 0 objects is a morphism $X \stackrel{e}{\rightarrow} Y$ satisfying weak right cancellation: if $Y \stackrel{g}{\rightarrow} Z$ is a morphism such that $g \circ e=0$, then $g=0$. The notion of weak epimorphism, as well as the dual notion of weak monomorphism (a morphism satisfying weak left cancellation), arises rather naturally in the pointed homotopy category $\mathscr{H}$ of topological spaces (compare [R]; in [R], the objects in $\mathscr{H}$ are taken to be path-connected $\mathrm{CW}$-spaces, but this restriction is unncessary here) and our purpose here is to compare this notion with the more traditional notion of homotopy-epimorphism ( = epimorphism in $\mathscr{H}$ ).

A study of the comparison between weak monomorphisms and monomorphisms in homotopy theory was carried out by Ganea [G] who, in particular, answered a number of questions raised in Hilton's notes [H2]. An additional problem hinted at in [H2; p. 180] is settled here; we show that weak epimorphisms in $\mathscr{H}$ are, in general, genuinely weaker than epimorphisms in $\mathscr{H}$.

Before proceeding with the details, a few remarks linking the notions of (weak) epimorphism and (weak) monomorphism in $\mathscr{H}$ may be in order. By definition, a (weak) monomorphism $X \rightarrow Y$ induces a (weak) monomorphism of pointed morphism sets $[W, X] \rightarrow[W, Y]$ for each $W$ in $\mathscr{H}$. Similarly, a (weak) epimorphism $X \rightarrow Y$ induces a (weak) monomorphism of pointed morphism sets $[Y, Z] \rightarrow[X, Z]$ for each $Z$ in $\mathscr{H}$. Sharper statements are provided by the following. 
Proposition 1.1. (i) $A$ (weak) monomorphism $X \rightarrow Y$ induces $a$ (weak) monomorphism $X^{W} \rightarrow Y^{W}$ of pointed mapping spaces for each $W$ in $\mathscr{H}$.

(ii) $A$ (weak) epimorphism $X \rightarrow Y$ induces a (weak) monomorphism $Z^{Y} \rightarrow Z^{X}$ of pointed mapping spaces for each $Z$ in $\mathscr{H}$.

Proposition 1.1 (ii) provides a method for creating (weak) monomorphisms out of (weak) epimorphisms. However, this half of Proposition 1.1 does not dualize and we merely have

Proposition 1.1'. A (weak) epimorphism $X \rightarrow Y$ induces a (weak) epimorphism $X \wedge W \rightarrow Y \wedge W$ of smash products.

It thus appears unlikely that Ganea's examples of weak monomorphisms which are not monomorphisms can be somehow transformed into examples of weak epimorphisms which are not epimorphisms (all in $\mathscr{H}$ ). In any event, Ganea's examples and the examples presented in $\S 2$ and $\S 3$ below utilize radically different techniques. Ganea requires very detailed knowledge of the homotopy groups of spheres to establish that his examples, though clearly weak monomorphisms, are not monomorphisms. On the other hand, our examples are based on the properties of a certain group introduced by Higman [H1] and further studied by Dyer and Vasquez [DV].

2. In most categories with 0 objects (for example, the category of pointed sets, or the category $\mathscr{H}[\mathbf{G}])$ the notions of weak monomorphism and monomorphism differ, but in the category $\mathscr{G}$ of groups, they clearly coincide. However, the notions of weak epimorphism and epimorphism do not coincide in $\mathscr{G}$. In fact, we have the following intrinsic characterization of weak epimorphisms in $\mathscr{G}([\mathbf{R} ; \S 3])$.

Proposition 2.1. A group homomorphism $\phi: G_{1} \rightarrow G_{2}$ is a weak epimorphism if and only if the normal closure of $\phi G_{1}$ in $G_{2}$ is all of $G_{2}$.

It is also possible to intrinsically characterize weak epimorphisms and weak monomorphisms in $\mathscr{H}$.

Proposition 2.2. (i) $X \stackrel{f}{\rightarrow} Y$ is a weak epimorphism in $\mathscr{H}$ if and only if $Y$ is contractible in the homotopy-cofiber of $f$.

(ii) $X \stackrel{f}{\rightarrow} Y$ is a weak monomorphism in $\mathscr{H}$ if and only if the homotopyfiber of $f$ is contractible in $X$. 
Now any group homomorphism $\phi: G_{1} \rightarrow G_{2}$ gives rise to a unique morphism $f: K\left(G_{1}, 1\right) \rightarrow K\left(G_{2}, 1\right)$ in $\mathscr{H}$, where $K\left(G_{i}, 1\right)$ is an EilenbergMacLane complex and where $f_{*}: \pi_{1} K\left(G_{1}, 1\right) \rightarrow \pi_{1} K\left(G_{2}, 1\right)$ coincides with $\phi$. But knowing that $\phi$ is a (weak) epimorphism in $\mathscr{G}$ does not allow us to infer that $f$ is a (weak) epimorphism in $\mathscr{H}$. Nevertheless, if $G_{2}$ is chosen carefully, we may in fact make this latter inference and thus arrive at a suitable example.

To this end, let $H$ be the Higman group [H1] with presentation.

$$
H=\left\langle a, b, c, d ; b a b^{-1}=a^{2}, c b c^{-1}=b^{2}, d c d^{-1}=c^{2}, a d a^{-1}=d^{2}\right\rangle
$$

According to [H1], if $N$ is a maximal normal subgroup of $H, N \neq H$, the quotient group $H / N$ is an infinite simple group. Now let $x$ be an element of $H$ not in $N$ and take $H_{0}$ to be the subgroup of $H$ generated by $N$ and $x$. Further, let $\phi: H_{0} \hookrightarrow H$ be the inclusion homomorphism and $f$ : $K\left(H_{0}, 1\right) \rightarrow K(H, 1)$ the induced morphism of Eilenberg-MacLane complexes.

THEOREM 2.1. $f: K\left(H_{0}, 1\right) \rightarrow K(H, 1)$ is a weak epimorphism but not an epimorphism in $\mathscr{H}$.

Proof. As $H_{0} / N$ is a cyclic subgroup of $H / N$ (generated by the coset $x N$ ) and as $H / N$ is infinite simple, it follows that $H_{0} / N$ is a proper subgroup of $H / N$. Thus $\phi: H_{0} \hookrightarrow H$ is not an epimorphism in $\mathscr{G}$ and so ([HR; Prop. 1]) $f: K\left(H_{0}, 1\right) \rightarrow K(H, 1)$ is not an epimorphism in $\mathscr{H}$. By the maximality of $N$ and Proposition 2.1, $\phi$ is a weak epimorphism in $\mathscr{G}$. To see that $f$ is a weak epimorphism in $\mathscr{H}$, let $g: K(H, 1) \rightarrow Z$ be a morphism with $g \circ f=0$. As $g_{*} \circ f_{*}=0: \pi_{1} K\left(H_{0}, 1\right) \rightarrow \pi_{1} Z$ and as $f_{*}=\phi$ is a weak epimorphism in $\mathscr{G}$, we see that $g_{*}=0$, so that $g$ lifts (uniquely) to $\tilde{g}: K(H, 1) \rightarrow \tilde{Z}, \tilde{Z}$ the universal cover of $Z$. (Since $K(H, 1)$ is a $C W$-space, $Z$ may be assumed, without loss of generality, to admit a universal cover. Alternatively, using Proposition 2.2 (i), $g$ may be taken to be the canonical map from $K(H, 1)$ to the homotopy-cofiber of $f$ and the latter is 1-connected by van Kampen's theorem.) But the $k$-dimensional obstruction to $\tilde{g}=0$ lies in the (constant coefficient) cohomology group $H^{k}\left(K(H, 1) ; \pi_{k} \tilde{Z}\right), k \geq 2$. As $H$ is acyclic and geometrically finite ([DV; $\S 4]$ ), these obstructions vanish, $\tilde{g}=0$, and hence $g=0$.

REMARK. While the model of $K(H, 1)$ constructed in [DV] is a finite 2-dimensional complex, we do not know whether $K\left(H_{0}, 1\right)$ has the homotopy type of a finite complex. Of course, since it may be viewed as a covering space of $K(H, 1), K\left(H_{0}, 1\right)$ certainly has the homotopy type of a 2-dimensional complex. 
3. The example given in this section requires a more elaborate argument than that of $\S 2$ but it yields a somewhat stronger result. To explain, we recall that a morphism $X \stackrel{f}{\rightarrow} Y$ in $\mathscr{H}$ gives rise to a 'cofibration sequence'

$$
X \stackrel{f}{\rightarrow} Y \stackrel{q}{\rightarrow} C \stackrel{s}{\rightarrow} \Sigma X \stackrel{\Sigma f}{\rightarrow} \Sigma Y \rightarrow \cdots
$$

Here, $q$ is the canonical morphism from $Y$ to the homotopy-cofiber of $f, s$ is the 'connecting' morphism from $C$ to the reduced suspension of $X$, and so on.

Theorem 2.1 shows that $f$ need not be an epimorphism even if $q=0$. Theorem 3.1 below will show that $q$ need not be an epimorphism even if $s=0$; in other words, even if a morphism, when viewed as a cofibration, is co-induced, it may fail to be an epimorphism while being a weak epimorphism. Since Hilton [H2; p. 180] has shown that $s$ is an epimorphism if and only if $\Sigma f=0$, it follows that Theorem 3.1 provides an example which is, in some sense, optimal.

We return now to the space $K(H, 1)$ studied in $\S 2$. If $W$ is the 4-fold wedge of circles $S^{1} \vee S^{1} \vee S^{1} \vee S^{1}$, there is a morphism $W \stackrel{f}{\rightarrow} W$ derived from the relators in the presentation of $H$ given in $\$ 2$ and according to [DV; §4] the homotopy-cofiber of $f$ is a $K(H, 1)$. Thus we have a cofibration sequence

$$
W \stackrel{f}{\rightarrow} W \stackrel{q}{\rightarrow} K(H, 1) \stackrel{s}{\rightarrow} \Sigma W \stackrel{\Sigma f}{\rightarrow} \Sigma W \rightarrow \cdots
$$

THEOREM 3.1. $q: W \rightarrow K(H, 1)$ is a weak epimorphism but not an epimorphism in $\mathscr{H}$.

Proof. The proof that $q$ is a weak epimorphism is precisely the same as in Theorem 2.1. However, as $q_{*}: \pi_{1} W \rightarrow \pi_{1} K(H, 1)$, it is not so simple to deduce that $q$ is not an epimorphism.

For that purpose, recall ([DV; §4]) that there exists an $H$-module $M$ such that the (twisted) cohomology group $H^{2}(K(H, 1) ; M)$ is non- 0 . The homomorphism $H \stackrel{\alpha}{\rightarrow}$ Aut $M$ describing this $H$-module structure gives rise to a sectioned fibration $E \rightarrow K(H, 1)$ with fiber the Eilenberg-MacLane space $K(M, 2)$ and a twisted version of the usual representation theorem ([S]) identifies $H^{2}(K(H, 1) ; M)$ with the homotopy classes (= vertical homotopy classes) of sections of this fibration. Let then $u$, $v \in H^{2}(K(H, 1) ; M), u \neq v$ and view $u, v$ as morphisms from $K(H, 1)$ to 
$E$. Pulling back via $q$ yields a morphism of fibrations

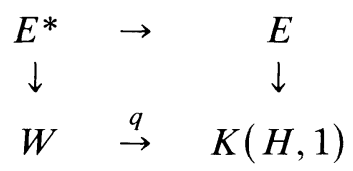

and $u, v$ pull back to sections $u^{*}, v^{*}$ of the induced fibration $E^{*} \rightarrow W$. But $u^{*}=v^{*}$ since each may be viewed as an element of $H^{2}\left(W ; q^{*} M\right)=0$; here $q^{*} M$ denotes the module over the free group on 4 generators associated to the homomorphism

$$
\pi_{1} W \stackrel{q_{*}}{\rightarrow} \pi_{1} K(H, 1)=H \stackrel{\alpha}{\rightarrow} \text { Aut } M .
$$

It follows then that $u \circ q=v \circ q$, hence that $q$ is not an epimorphism.

Remark. Since $H^{2}(K(H, 1) ; M)=0$ for any $H$-module $M$ which is finitely generated as an abelian group ([DV; $\$ 4])$, obstruction theory shows that $q$ is an epimorphism in the full subcategory of $\mathscr{H}$ whose objects are pointed spaces with finitely generated second homotopy groups. In contrast, the morphism $f: K\left(H_{0}, 1\right) \rightarrow K(H, 1)$ of $\S 2$ is plainly not an epimorphism in this subcategory nor, indeed, in any full subcategory of $\mathscr{H}$ containing the Eilenberg-MacLane spaces $K(G, 1), G$ a finitely generated group.

\section{REFERENCES}

[DV] E. Dyer and A. T. Vasquez, Some small aspherical spaces, J. Australian Math. Soc., 16 (1973), 332-352.

[G] T. Ganea, On monomorphisms in homotopy theory, Topology, 6 (1967), 149-152.

[H1] G. Higman, A finitely generated infinite simple group, J. London Math. Soc., 26 (1951), 61-64.

[H2] P. Hilton, Homotopy Theory and Duality, Notes on Mathematics and its Applications, Gordon and Breach, 1965.

[HR] P. Hilton and J. Roitberg, Note on epimorphisms and monomorphisms in homotopy theory, Proc. Amer. Math. Soc., 90 (1984), 316-320.

[R] J. Roitberg, Residually finite, Hopfian and co-Hopfian spaces, In: Conference on Algebraic Topology in Honor of Peter Hi Hon (R. Piccinini and D. Sjerve, Editors) Contemporary Mathematics, 37 (1985), 131-144.

[S] J. Siegel, Cohomology and homology theories for categories of principal G-bundles, Trans. Amer. Math. Soc., 120 (1965), 428-437.

Received May 29, 1984 and in revised form August 6, 1984.

Hunter College

CUNY

NEW YORK, NY 10021 



\section{PACIFIC JOURNAL OF MATHEMATICS EDITORS}

V. S. VARADARAJAN (Managing Editor)
University of California
Los Angeles, CA 90024
HEBERT CLEMENS
University of Utah
Salt Lake City, UT 84112
CHARLES R. DEPRIMA
California Institute of Technology
Pasadena, CA 91125

\section{R. FINN}

Stanford University

Stanford, CA 94305

HeRmanN FlaschKa

University of Arizona

Tucson, AZ 85721

RAMESH A. GANGOLli

University of Washington

Seattle, WA 98195

ROBION KIRBY

University of California

Berkeley, CA 94720

\section{C. MoOre}

University of California

Berkeley, CA 94720

H. SAMELSON

Stanford University

Stanford, CA 94305

HAROLD STARK

University of California, San Diego

La Jolla, CA 92093

ASSOCIATE EDITORS
R. ARENS
E. F. BECKENBACH
B. H. NeUmanN
F. WOLF
K. Yoshida (1906-1982)

\section{SUPPORTING INSTITUTIONS}

UNIVERSITY OF ARIZONA

UNIVERSITY OF BRITISH COLUMBIA

CALIFORNIA INSTITUTE OF TECHNOLOGY

UNIVERSITY OF CALIFORNIA

MONTANA STATE UNIVERSITY

UNIVERSITY OF NEVADA, RENO

NEW MEXICO STATE UNIVERSITY

OREGON STATE UNIVERSITY
UNIVERSITY OF OREGON

UNIVERSITY OF SOUTHERN CALIFORNIA

STANFORD UNIVERSITY

UNIVERSITY OF HAWAII

UNIVERSITY OF TOKYO

UNIVERSITY OF UTAH

WASHINGTON STATE UNIVERSITY

UNIVERSITY OF WASHINGTON 


\section{Pacific Journal of Mathematics}

\section{Vol. 121, No. $1 \quad$ November, 1986}

Om P. Agrawal, Douglas Napier Clark and Ronald George Douglas,

Invariant subspaces in the polydisk $\ldots \ldots \ldots \ldots \ldots \ldots \ldots \ldots \ldots \ldots$

Christoph Bandt and Gebreselassie Baraki, Metrically invariant measures

on locally homogeneous spaces and hyperspaces $\ldots \ldots \ldots \ldots \ldots \ldots \ldots$

Marcy Mason Barge, Horseshoe maps and inverse limits ..............29

Russell Gene Bilyeu, Robert Richard Kallman and Paul Weldon Lewis,

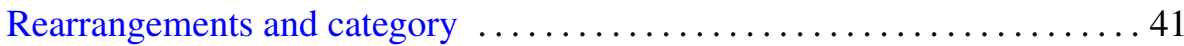

Jean Bourgain, A problem of Douglas and Rudin on factorization . . .......47

Hernan Cendra, A normal form and integration in finite terms for a class of

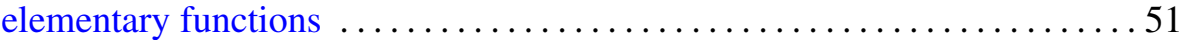

Ky Fan, The angular derivative of an operator-valued analytic function . . . . 67

Gerhard Gierz, On the Dunford-Pettis property of function modules of

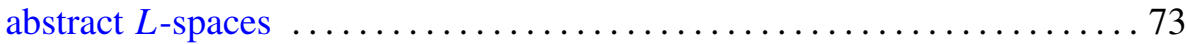

Gabriel Katz, On polynomial generators in the algebra of complex functions

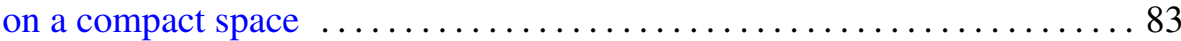

Ridgley Lange, Duality and asymptotic spectral decompositions $\ldots \ldots \ldots . .93$

Anthony To-Ming Lau and Peter F. Mah, Quasinormal structures for certain spaces of operators on a Hilbert space ................... 109

R. Daniel Mauldin, Correction: "The set of continuous nowhere differentiable functions"

Alan Harvey Mekler and Saharon Shelah, $\omega$-elongations and Crawley's problem

Alan Harvey Mekler and Saharon Shelah, The solution to Crawley's problem

Richard Rochberg, Deformation of uniform algebras on Riemann surfaces

Joseph Roitberg, On weak epimorphisms in homotopy theory

Jesús M. Ruiz, A remark on fields with the dense orbits property

Henry Wente, Counterexample to a conjecture of H. Hopf

David G. Wright, Rigid sets in $E^{n}$ 